\title{
Task Force - «Naturheilkunde und Komplementärmedizin in medizinischen Leitlinien »
}

\author{
Jost Langhorst \\ Department of Internal and Integrative Medicine, Kliniken Essen-Mitte, Faculty of Medicine, University of Duisburg-Essen, Essen, Germany
}

\section{Schlüsselwörter \\ Naturheilkunde · Komplementärmedizin · Medizinische Leitlinien · AWMF · Gesellschaft für Phytotherapie . Nationale Versorgungsleitlinie · S3-Leitlinien}

\section{Zusammenfassung}

Im Rahmen der aktuellen Wissenschaftskultur stellt das Bestreben, die evidenzbasierte Naturheilkunde in die Leitlinien zu integrieren, eine besondere Herausforderung dar. In Deutschland werden medizinische Leitlinien primär von den wissenschaftlichen medizinischen Fachgesellschaften und deren Dachgesellschaft, der Arbeitsgemeinschaft der Wissenschaftlichen Medizinischen Fachgesellschaften e.V. (AWMF), entwickelt und verbreitet. Bisher wird das große klinische Potential von Naturheilkunde und Komplementärmedizin an vielen Stellen nur unzureichend berücksichtigt. Mit der Aufnahme der Gesellschaft für Phytotherapie (GPT) in die AWMF wurde im Jahr 2013 ein neues Kapitel in der Leitlinienarbeit der von der Rut- und Klaus-BahlsenStiftung geförderten Arbeitsgruppe «Naturheilkunde und Komplementärmedizin in medizinischen Leitlinien" aufgeschlagen. Die Bestrebung, Naturheilkunde und Komplementärmedizin fester in der konventionellen medizinischen Versorgung in Deutschland zu etablieren, bekommt damit weiter Auftrieb. Durch die Förderung der Rut- und Klaus-Bahlsen-Stiftung wurden die Mitarbeit oder Unterstützung bei bisher mehr als 20 nationalen und internationalen Leitlinien, die Erstellung von 8 Cochrane-Reviews, 4 davon federführend, und von 40 systematischen Reviews und Meta-Analysen in internationalen Peer-Review-Zeitschriften möglich. Erst durch die konsequente Ausweitung und Vollendung der begonnenen Arbeit wird eine Rückentwicklung (und Rückabwicklung) im Feld der Leitlinienarbeit unmöglich gemacht und damit die Anerkennung von Naturheilkunde und Komplementärmedizin im Fächerkanon weiter gestärkt.

(c) 2016 S. Karger GmbH, Freiburg

\author{
Keywords \\ Complementary and Alternative Medicine (CAM) . \\ Medical guidelines - AWMF . Society for Phytotherapy . \\ German guideline $\cdot$ S3-guidelines
}

\section{Summary}

In the context of current scientific culture, the implementation of evidence-based complementary and integrative medicine in the medical guidelines represents a special challenge. In Germany, medical guidelines are developed by scientific medical societies and their coordinating organization, the Arbeitsgemeinschaft der Wissenschaftlichen Medizinischen Fachgesellschaften e.V. (AWMF). So far, the great clinical potential of complementary and integrative medicine is not sufficiently considered in many guidelines. With the acceptance of the Society of Phytotherapy (GPT) as full member in the AWMF in 2013, a new chapter in the guidelines work funded by the Rut- and Klaus-Bahlsen-Foundation was opened. The aim to implement complementary and integrative treatment approaches in the conventional medical care in Germany, thus further gets a boost. Promoted by the Rut- and KlausBahlsen-Foundation, the work group has participated or assisted in more than 20 national and international medical guidelines and has created 8 Cochrane reviews, 4 of these in leading responsibility, as well as 40 systematic reviews and meta-analyzes in international peer-reviewed journals. The aim for the future is to further strengthen the recognition and acceptance of naturopathy and complementary medicine in the medical guidelines and the health system.

\section{KARGER \\ Fax +497614520714

\section{() 2016 S. Karger GmbH, Freiburg}

$1661-4119 / 16 / 0233-0170 \$ 39.50 / 0$
Prof. Dr. Jost Langhorst

Department of Internal and Integrative Medicine, Kliniken Essen-Mitte Faculty of Medicine, University of Duisburg-Essen Am Deimelsberg 34a, 45276 Essen, Germany j.langhorst@kliniken-essen-mitte.de 


\section{Naturheilkunde und Komplementärmedizin in Deutschland}

Der Naturheilkunde kommt in der medizinischen Versorgung der Bevölkerung in Deutschland große Bedeutung zu. So geben $70 \%$ der Bevölkerung über 16 Jahren in Deutschland an, eigene Erfahrungen mit Naturheilmitteln zu haben [1]. Darüber hinaus sind Naturheilkunde und Komplementärmedizin ein großes und relevantes klinisches Querschnittsfach. In Deutschland haben nahezu 70000 Ärztinnen und Ärzte eine zertifizierte Zusatzqualifikation in Naturheilverfahren, Physikalischer Therapie und Balneologie, Manueller Medizin, Homöopathie oder Akupunktur [2].

Allerdings bildet sich diese Situation nicht adäquat in den Bereichen berufspolitischer Machtinstrumente wie der öffentlichen Ausbildungs- und Forschungsförderung oder der Gremienarbeit ab: Obwohl die Naturheilkunde seit 2003 Pflichtfach in der medizinischen Ausbildung (QB12) in Deutschland ist, gibt es, anders als z.B. in der Schweiz, keine öffentlich geförderten Lehrstühle für Naturheilkunde oder Integrative Medizin. Die großen öffentlichen Förderer wie die Deutsche Forschungsgemeinschaft und das Bundesministerium für Bildung und Forschung haben, anders als z.B. in den USA, keinen eigenen Forschungs- und Förderbereich für Naturheilkunde oder Komplementärmedizin. Bezeichnend ist außerdem, dass Naturheilkunde und Komplementärmedizin in wichtigen berufspolitischen Gremien über Jahre nicht vertreten waren bzw. seit Jahren nicht vertreten sind. So sind in der Arbeitsgemeinschaft der Wissenschaftlichen Medizinischen Fachgesellschaften (AWMF), die seit 1995 für die Erstellung der Leitlinien die Verantwortung trägt, derzeit 171 wissenschaftliche Fachgesellschaften aus allen Bereichen der Medizin zusammengeschlossen. Bis 2013 war allerdings keine Gesellschaft aus dem Bereich der Naturheilkunde oder Komplementärmedizin Mitglied, was weitreichende Folgen für deren Repräsentation in den AWMF-Leitlinien hatte. Nur durch Zufall, durch gewogene Vertreter anderer Fachgesellschaften, war dieser Bereich in den Leitlinien überhaupt vertreten - vor allem war er aber nicht vertreten.

\section{Medizinische Leitlinien}

Medizinische Leitlinien sind systematisch entwickelte Entscheidungshilfen für Ärzte zu medizinischen Themen. Sie haben den Zweck, die wissenschaftliche Evidenz und die vorhandene Erfahrung zu medizinischen Themen zusammenzufassen, gegensätzliche Standpunkte zu klären und das aktuelle Vorgehen der Wahl zu definieren. Medizinische Leitlinien existieren seit den 90erJahren des 20. Jahrhunderts. Obwohl bereits zuvor die Notwendigkeit deutlich geworden war, die neuen und erweiterten Erkenntnisse in die medizinische Versorgung einzubinden und zu standardisieren, beinhalteten die Empfehlungen bis dahin vor allem Expertenmeinungen. Das ständig wachsende Wissen führt dazu, dass sich Empfehlungen für oder gegen Therapieverfahren ändern können. Aus diesem Grund haben Leitlinien eine kurze «Lebensdauer» von höchstens 5 Jahren und werden regelmäßig auf den neuesten
Stand gebracht. Zur Erstellung einer evidenzbasierten Leitlinie kommen in Deutschland die Vertreter der entsprechenden medizinischen Bereiche und Fachgesellschaften zusammen, um gemeinsam die aktuell existierenden wissenschaftlichen Informationen zu sammeln und auszuwerten. Der Entstehungsprozess einer solchen Leitlinie nimmt oftmals mehrere Jahre in Anspruch.

Leitlinien sind zu einem wichtigen Bestandteil des Qualitätsmanagements im Gesundheitswesen geworden, auch wenn sie in erster Linie «nur» Orientierungshilfen sind, von denen im Einzelfall abgewichen werden kann. Medizinische Leitlinien haben in den letzten Jahren zunehmend an Bedeutung für das öffentliche medizinische Interesse gewonnen, ihr Einfluss ist vielschichtig. Zum einen dienen sie den aktuell im Gesundheitssystem aktiven Ärztinnen und Ärzten als Orientierung. Bedeutend ist weiterhin der Einfluss auf die aktuelle Studenten- und somit die kommende Ärztegeneration, die sich häufig schon nicht mehr an Lehrbüchern, sondern an den aktuellen Leitlinien der AWMF im Internet orientiert. Deutsche Leitlinien richten sich dabei nicht nur an medizinische Experten; sie sind häufig in 2 Fassungen erhältlich, 1 für Mediziner und 1 kürzeren Fassung für Patienten und Angehörige. Außerdem werden die Leitlinien häufig, wenn auch im juristischen Sinne nicht bindend, bei rechtlichen Auseinandersetzungen zur Beurteilung der empfohlenen Versorgung berücksichtigt und können damit relevant für die Berufsausübung werden. Nicht zuletzt werden die Leitlinien zunehmend bei Entscheidungen der Kostenübernahme durch die Krankenkassen und in inhaltlichen Auseinandersetzungen mit dem Medizinischen Dienst als Argumentationsgrundlage genutzt.

«Medizinische Leitlinien sind systematisch entwickelte Feststellungen, um die Entscheidungen von Ärzten, Angehörigen anderer Gesundheitsberufe und Patienten über angemessene Gesundheitsversorgung für spezifische klinische Umstände zu unterstützen» [3].

In Hinblick auf die Leitlinienarbeit lagen Naturheilkunde und Komplementärmedizin über 18 Jahre lang im «Dornröschenschlaf». Dieser Zustand wurde durch das Engagement der Rut- und Klaus-Bahlsen-Stiftung nachhaltig verändert.

\section{Task Force - die Arbeitsgruppe «Naturheilkunde und Komplementärmedizin in medizinischen Leitlinien "}

Gefördert von der Rut- und Klaus-Bahlsen-Stiftung konstituierte sich im Jahr 2001 die Arbeitsgruppe «Naturheilkunde und Komplementärmedizin in medizinischen Leitlinien» mit dem Arbeitsschwerpunkt, naturheilkundliche und komplementärmedizinische Verfahren in medizinische Leitlinien zu integrieren. Das Ziel dieser Arbeit ist die Kooperation und gemeinsame Erstellung fundierter und wissenschaftlich hochwertiger Leitlinien mit Vertretern der konventionellen Medizin. Die systematische Aufarbeitung wissenschaftlicher Studien aus dem Bereich der Naturheilkunde und Komplementärmedizin ist nicht zuletzt vor dem Hintergrund der kontinuierlich zunehmenden Inanspruchnahme von 
Naturheilkunde und komplementärmedizinischen Heilverfahren von zentraler Bedeutung.

Ein Meilenstein der bisherigen Arbeit waren die Aufnahme der Gesellschaft für Phytotherapie (GPT) in die AWMF und die Ernennung von Prof. Jost Langhorst zum Beauftragten der GPT für Leitlinienarbeit im Jahr 2014. Hiermit konnte eine neue Ära in der Leitlinienarbeit der von der Rut- und Klaus-Bahlsen-Stiftung geförderten Task Force «Naturheilkunde und Komplementärmedizin in medizinischen Leitlinien» eingeleitet werden.

Aufgrund der zunehmend breiten Akzeptanz und des konstant hohen Bedarfs für eine hochqualifizierte Leitlinienarbeit im Bereich Naturheilkunde und Komplementärmedizin hat die Arbeitsgruppe auf nationaler und internationaler Ebene umfangreiche Beiträge zu zahlreichen Leitlinien geleistet und wichtige Grundlagen für deren Erstellung geschaffen. Durch die Gründung und Arbeit der Arbeitsgruppe ist es gelungen, einen bisher nicht besetzten Schlüsselbereich für die Naturheilkunde und Komplementärmedizin zu erschließen, der berufs- und gesundheitspolitisch von hoher Relevanz ist.

\section{Ergebnisse der Arbeit}

Wie wichtig die Arbeit ist, zeigen 2 Studien unserer Arbeitsgruppe, die die Berücksichtigung der Phytotherapie als einen zentralen Bereich der Naturheilkunde in den Leitlinien im Fokus haben: In 55 der zum Untersuchungszeitpunkt im März 2014 verfügbaren 128 S3-Leitlinien (42,9\%) mit potenziellen phytotherapeutischen Therapieoptionen wurden pflanzliche Arzneimittel nicht berücksichtigt. In 40 (31,3\%) der verfügbaren S3-Leitlinien wurde die Phytotherapie zwar genannt, von den insgesamt 204 Empfehlungen/Kommentaren zu Phytotherapeutika fielen 139 $(68,1 \%)$ allerdings negativ aus oder blieben ergebnisoffen. Hinweise zu Neben- und Wechselwirkungen fanden sich zu 70 der 204 Empfehlungen (34,3\%). Also lagen nur in weit weniger als der Hälfte der verfügbaren AWMF-S3-Leitlinien eine Recherche zu Phytotherapeutika und nur in 17 (13,3\%) positive Empfehlungen zur Phytotherapie vor [4].

In der zweiten Arbeit der Arbeitsgruppe zum Thema, die Sie in dieser Ausgabe der Forschenden Komplementärmedizin finden [5], wurde untersucht, inwieweit Neben- bzw. Wechselwirkungen von pflanzlichen Arzneimitteln in den verfügbaren S3-Leitlinien diskutiert werden. Im Januar 2015 wurden hierzu die verfügbaren 134 S3-Leitlinien analysiert. Zu diesem Zeitpunkt wiesen 27,6\% $(\mathrm{n}=37)$ insgesamt 194 Statements zur Phytotherapie auf. Hinweise zu Neben- und Wechselwirkungen fanden sich nur bei $28,4 \%$ der Statements $(n=55)$, die bei nur 13,9\% $(n=27)$ durch Literatur belegt waren. In vielen Fällen wiesen die Leitlinien unpräzise Begrifflichkeiten sowie eine unzureichende Systematik bei der Suche nach wissenschaftlicher Evidenz für die Beurteilung von Arzneipflanzen-Zubereitungen auf.

Durch die Förderung der Rut- und Klaus-Bahlsen-Stiftung wurden die Mitarbeit oder Unterstützung bei bisher mehr als 20 nationalen und internationalen Leitlinien, die Erstellung von 8
Cochrane-Reviews, 4 davon federführend, und von 40 systematischen Reviews und Meta-Analysen in internationalen PeerReview-Zeitschriften sowie über 44 weitere Publikationen möglich [6]. Diese Basisarbeit ist auch im internationalen Vergleich einzigartig. Auf der wachsenden Grundlage der seriösen Vorarbeiten ist es schon jetzt gelungen, zahlreiche naturheilkundliche und komplementärmedizinische Verfahren in bedeutenden Leitlinien mit positiven oder offenen Statements und Kommentaren zu implementieren. Ausgewählte Beispiele hierfür sind [7]:

- Phytotherapie bei Reizdarmsyndrom (S3), Colitis ulcerosa (S3), Obstipation (S2k), nichtspezifischem Rückenschmerz (NVL), Husten (Deutsche Gesellschaft für Allgemeinmedizin und Familienmedizin (DEGAM)) und Mammakarzinom (S1);

- Mind-Body-Medicine bei Colitis ulcerosa und Mammakarzinom;

- Meditative Bewegungstherapien (Yoga, Qi Gong, Tai Chi) bei Reizdarmsyndrom, Fibromyalgiesyndrom (S3), NVL, Unterbauchschmerz der Frau (S3) und Mammakarzinom;

- Akupunktur bei Morbus Crohn, Colitis ulcerosa, NVL, Fibromyalgiesyndrom, Unterbauchschmerz der Frau und Mammakarzinom;

- Bewegung bei Reizdarmsyndrom, Obstipation, NVL und Fibromyalgiesyndrom;

- Ernährung: Reizdarmsyndrom, Obstipation und Mammakarzinom;

- Probiotikatherapie bei Reizdarmsyndrom, Colitis ulcerosa und Obstipation;

- Homöopathie bei Mammakarzinom und Fibromyalgiesyndrom (Minderheitenvotum).

Neben einer großen Anzahl von Vorträgen auf nationalen und internationalen Kongressen zu diesem Thema war das wissenschaftliche Symposium am 4. Dezember 2015 in Essen zu «Naturheilkunde und Komplementärmedizin in medizinischen Leitlinien» ein besonderer Höhepunkt der Arbeit, die nicht zuletzt durch den intensiven Dialog mit der AWMF große Beachtung gefunden hat.

Die Kooperation mit relevanten Fachgesellschaften wie der DEGAM und der Deutschen Gesellschaft für Gastroenterologie, Verdauung und Stoffwechselerkrankungen (DGVS) sowie verstärkte Aktivitäten im Bereich onkologischer Leitlinien durch die Vertiefung der Kooperation mit dem Kompetenznetz «Komplementärmedizin in der Onkologie» ermöglichen die weitere Vertiefung des Engagements.

\section{Perspektive}

Erst jetzt, nach dem intensiven Engagement der Task Force seit 2011 und der konsequenten Qualifizierung der Mitarbeiter, sind wir in der Lage, den Einfluss für Naturheilkunde und Komplementärmedizin optimal zu gestalten, einzusetzen und kontinuierlich auszuweiten.

Ein wichtiges Indiz hierfür ist die erstmalige Mitarbeit im Jahr 2015 an 1 von 7 Nationalen Versorgungsleitlinien (NVL; [6]) - der 
NVL für unspezifischen Kreuzschmerz. Dabei ist es uns gelungen, wichtige Therapien aus dem Feld Naturheilkunde und Komplementärmedizin (Akupunktur, Phytotherapie (Weidenrinde) sowie meditative Bewegungsformen (Yoga, Tai Chi, Alexandertechnik)) $\mathrm{zu}$ positionieren. Im November 2015 erfolgte nun folgerichtig die Einladung zur Mitarbeit an der Überarbeitung der zweiten NVL, nämlich für Asthma bronchiale, im Februar 2016 die Einladung zur Mitarbeit an der NVL Koronare Herzkrankheit. Insgesamt hat das konsequente Engagement der Arbeitsgruppe im Bereich Leitlinien dazu geführt, dass die Nachfrage und das Angebot der Mitarbeit an strategisch relevanten Leitlinien auf allen Ebenen erheblich zugenommen haben. Weitere Beispiele für in Arbeit befindliche Leitlinien unter Beteiligung der Task Force sind aktuell die S3-Leitlinie zu Hepatitis B, die S3-Leitlinie zum Magenkarzinom, die S3-Leitlinie zu Schlafstörungen und die S2k-Leitlinie zur Gonarthrose.
Die Rut- und Klaus-Bahlsen-Stiftung hat durch ihr visionäres Engagement in der Leitlinienarbeit ein grundlegendes Umdenken in der Szene und in der Medizin vorbereitet. Entscheidend für den langfristigen und nachhaltigen Erfolg ist es nun, den begonnenen Prozess stetig voranzutreiben und auszuweiten.

Erst durch die konsequente Ausweitung und Vollendung der begonnenen Arbeit wird eine Rückentwicklung (und Rückabwicklung) im Feld der Leitlinienarbeit unmöglich gemacht und damit die Anerkennung von Naturheilkunde und Komplementärmedizin im Fächerkanon weiter gestärkt.

\section{Disclosure Statement}

Der Autor erklärt hiermit, dass keine Interessenskonflikte in Bezug auf die vorliegende Arbeit bestehen.

\section{Literatur}

1 Institut für Demoskopie Allensbach: Naturheilmittel 2010. Ergebnisse einer bevölkerungsrepräsentativen Befragung. Allensbach, Institut für Demoskopie Allensbach, 2010,www.ifd-allensbach.de/uploads/tx_studies/ 7528_Naturheilmittel_2010.pdf (letzter Abruf 30. Mai 2016).

2 Statistisches Bundesamt: Das Informationssystem der Gesundheitsberichterstattung des Bundes, Stand 11 Juni 2015, www.gbe-bund.de.
3 Lorenz W: 1. Rundbrief: Was sind Leitlinien? Rundbrief der AWMF, 1999, www.awmf.org/fileadmin/user_ upload/Leitlinien/Werkzeuge/Publikationen/rb1.pdf (letzter Abruf 30. Mai 2016).

4 Klose P, Kraft K, Cramer H, et al: Phytotherapie in den medizinischen S3-Leitlinien der Arbeitsgemeinschaft der Wissenschaftlichen Medizinischen Fachgesellschaften - eine systematische Übersichtsarbeit. Forsch Komplementärmed Klass Naturheilkd 2014;21:388-400.
Klose P, Kraft K, Lauche R, et al: Berücksichtigung von Wechsel- und Nebenwirkungen bei pflanzlichen Arzneimitteln in deutschen S3-Leitlinien der AWMF. Forsch Koplementmed 2016;23:154-164.

6 www.leitlinien.de/nvl (letzter Abruf 30. Mai 2016).

7 www.awmf.org/leitlinien/aktuelle-leitlinien.html (letzter Abruf 30. Mai 2016). 